\title{
Convergence Results for Piecewise Linear Quadratures for Cauchy Principal Value Integrals
}

\author{
By Philip Rabinowitz
}

\begin{abstract}
Conditions on $k$ and $f$ are given for the pointwise and uniform convergence to the Cauchy principal value integral

$$
\int_{-1}^{1} \frac{k(x) f(x)}{x-\lambda} d x, \quad-1<\lambda<1
$$

of a sequence of integrals of piecewise linear approximations to $f(x)$ or $g_{\lambda}(x)=$ $(f(x)-f(\lambda)) /(x-\lambda)$. The important special case, $k(x)=(1-x)^{\alpha}(1+x)^{\beta}$, is considered in detail.
\end{abstract}

1. Introduction. In a recent paper, Gerasoulis [3] proposed an algorithm for evaluating Cauchy principal value integrals of the form

$$
I(w f ; \lambda)=f_{-1}^{1} w(x) \frac{f(x)}{x-\lambda} d x, \quad-1<\lambda<1,
$$

where $w$ is the Jacobi weight function

$$
w(x)=(1-x)^{\alpha}(1+x)^{\beta}, \quad \alpha, \beta>-1 .
$$

In this algorithm, $f$ is approximated by a piecewise linear function $f_{n}$ and $I\left(w f_{n} ; \lambda\right)$ is evaluated exactly. The use of $f_{n}$ as an approximating function allows us to concentrate the evaluation points in subintervals of $J=[-1,1]$ where $f$ is not smooth. In his paper Gerasoulis asserts that if $f \in C^{1}[J]$, then $I\left(w f_{n} ; \lambda\right)$ converges to $I(w f ; \lambda)$ uniformly for all $\lambda \in(-1,1)$, and refers to Stewart [6] for the proof. The uniform convergence is important in the numerical solution of Cauchy singular integral equations by quadrature methods.

In this paper, we shall improve on this result by giving weaker conditions on $f$ which insure the uniform convergence of $I\left(w f_{n} ; \lambda\right)$ to $I(w f ; \lambda)$. We shall also study pointwise convergence for the more general situation

$$
I(k f ; \lambda)=f_{-1}^{1} k(x) \frac{f(x)}{x-\lambda} d x, \quad-1<\lambda-1,
$$

where $k$ is an arbitrary function subject to certain conditions insuring that (3) exists for some class of functions $f$ and some or all $\lambda$ in $(-1,1)$. Finally, we shall study an alternative approach to the evaluation of (3) which also allows us to distribute evaluation points as we see fit. In this approach, we write (3) as

$$
I\left(k f ; \lambda j=\int_{-1}^{1} k(x) g_{\lambda}(x) d x+f(\lambda) I(k ; \lambda),\right.
$$

Received November 12, 1987.

1980 Mathematics Subject Classification (1985 Revision). Primary 65D30.

Key words and phrases. Cauchy principal value integrals, piecewise linear approximation, product integration, Jacobi weight function. 
where

$$
g_{\lambda}(x)=g(x ; \lambda)= \begin{cases}\frac{f(x)-f(\lambda)}{x-\lambda}, & x \neq \lambda \\ f^{\prime}(\lambda), & x=\lambda, f^{\prime}(\lambda) \text { exists } \\ 0, & \text { otherwise }\end{cases}
$$

We then approximate $g_{\lambda}(x)$ by a piecewise linear function $g_{\lambda n}(x)$ and integrate exactly. This amounts to piecewise linear product integration of $g_{\lambda}$ with respect to $k$. We shall show that subject to certain conditions on $f$ and $k$, the product integral

$$
\int_{-1}^{1} k(x) g_{\lambda n}(x) d x \rightarrow \int_{-1}^{1} k(x) g_{\lambda}(x) d x \quad \text { as } n \rightarrow \infty
$$

either pointwise or uniformly in $\lambda \in(-1,1)$ depending upon the conditions we impose.

2. Preliminary Results. Our first task is to insure that (3) exists. To this end, following [1], we define, for any interval $I$ of length $l(I)$, a subclass of $C(I)$ of functions of Dini type, $D T(I)$, by

$$
D T(I)=\left\{h: \int_{0}^{l(I)} \omega_{I}(h ; t) t^{-1} d t<\infty\right\}
$$

where $\omega_{I}(h ; t)$ is the modulus of continuity of $h$ in $I$,

$$
\omega_{I}(h ; t)=\sup _{\substack{\left|x_{1}-x_{2}\right| \leq t \\ x_{1}, x_{2} \in I}}\left|h\left(x_{1}\right)-h\left(x_{2}\right)\right| .
$$

We shall write $\omega(h ; t)$ for $\omega_{J}(h ; t)$. We now have that if $\lambda \in(-1,1)$, and if for some $\delta>0$ such that the closed subinterval $N_{\delta}(\lambda)=[\lambda-\delta, \lambda+\delta] \subset J, k \in$ $D T\left(N_{\delta}(\lambda)\right) \cap L_{1}(J)$ and $f \in D T\left(N_{\delta}(\lambda)\right) \cap R(J)$, where $R(J)$ is the set of bounded Riemann-integrable functions on $J$, then (3) exists. This follows by writing

(7) $I(k f ; \lambda)=\int_{-1}^{1} k(x) g_{\lambda}(x) d x+f(\lambda) \int_{-1}^{1} \frac{k(x)-k(\lambda)}{x-\lambda} d x+f(\lambda) k(\lambda) f_{-1}^{1} \frac{d x}{x-\lambda}$.

Since

$$
f_{-1}^{1} \frac{d x}{x-\lambda}=\log \frac{1-\lambda}{1+\lambda}
$$

the hypotheses on $k$ and $f$ ensure that the first two integrals in (7) are finite. In particular, since $w \in D T(-1,1) \cap L_{1}(J)$, it follows that (1) exists for all $\lambda \in(-1,1)$ provided that $f \in D T(-1,1) \cap R(J)$.

We now consider an arbitrary partition of $J$,

$$
-1=t_{0}<t_{1}<\cdots<t_{n-1}<t_{n}=1,
$$

and define for any function $h$ on $J$ the piecewise linear approximation $h_{n}$ given by

$$
h_{n}(x)=h\left(t_{i}\right)+\frac{\left(x-t_{i}\right)}{d_{i}}\left(h\left(t_{i+1}\right)-h\left(t_{i}\right)\right), \quad x \in\left[t_{i}, t_{i+1}\right]
$$

where $d_{i}=t_{i+1}-t_{i}, i=0, \ldots, n-1$. If we define

$$
D_{n}=\max _{0 \leq i \leq n-1} d_{i}
$$


we shall assume that $D_{n} \rightarrow 0$ as $n \rightarrow \infty$. Defining

$$
r_{n}(x)=h(x)-h_{n}(x),
$$

we have that if $h \in C(J)$, then

$$
\left\|r_{n}\right\|=\max _{x \in J}\left|r_{n}(x)\right| \leq \omega\left(h ; D_{n}\right) .
$$

This follows since by Newton's interpolation formula with divided differences, if $x \in\left[t_{i}, t_{i+1}\right]$ then

$$
\begin{aligned}
h(x)-h_{n}(x) & =\left(x-t_{i}\right)\left(x-t_{i+1}\right) h\left[t_{i}, t_{i+1}, x\right] \\
& =-\left(x-t_{i}\right)\left(x-t_{i+1}\right)\left(h\left[t_{i}, x\right]-h\left[t_{i+1}, x\right]\right) / d_{i} \\
& =-\frac{\left(x-t_{i}\right)\left(x-t_{i+1}\right)}{d_{i}}\left[\frac{h(x)-h\left(t_{i}\right)}{x-t_{i}}-\frac{h(x)-h\left(t_{i+1}\right)}{x-t_{i+1}}\right] \\
& =-d_{i}^{-1}\left[\left(x-t_{i+1}\right)\left(h(x)-h\left(t_{i}\right)\right)-\left(x-t_{i}\right)\left(h(x)-h\left(t_{i+1}\right)\right)\right] .
\end{aligned}
$$

Hence

$$
\left|h(x)-h_{n}(x)\right| \leq d_{i}^{-1} \omega\left(h ; d_{i}\right)\left(\left|x-t_{i+1}\right|+\left|x-t_{i}\right|\right)=\omega\left(h ; d_{i}\right) \leq \omega\left(h ; D_{n}\right),
$$

where we have used the monotonicity of $\omega(h ; t)$. In addition, we have that

$$
\omega\left(h_{n} ; t\right) \leq 5 \omega(h ; t)
$$

which implies that

$$
\omega\left(r_{n} ; t\right) \leq 6 \omega(h ; t) .
$$

To show (10), we consider two cases:

Case 1. $x, x+\delta \in\left[t_{i}, t_{i+1}\right]$.

Then $\left|h_{n}(x+\delta)-h_{n}(x)\right|=\left|\delta\left(h\left(t_{i+1}\right)-h\left(t_{i}\right)\right) / d_{i}\right| \leq\left(\delta / d_{i}\right) \omega\left(h ; d_{i}\right)$. We now show that $\left(\delta / d_{i}\right) \omega\left(h ; d_{i}\right) \leq 2 \omega(h ; \delta)$. Since

$$
\begin{gathered}
\omega(h ; n \delta) \leq n \omega(h ; \delta), \\
\omega\left(h ; d_{i}\right) \leq \omega\left(h ;\left\lceil d_{i} / \delta\right\rceil \delta\right) \leq\left\lceil d_{i} / \delta\right\rceil \omega(h ; \delta) \leq 2\left(d_{i} / \delta\right) \omega(h ; \delta),
\end{gathered}
$$

where the ceiling function $\lceil x\rceil$ denotes the smallest integer $\geq x$. Thus, in this case, $\omega\left(h_{n} ; \delta\right) \leq 2 \omega(h ; \delta)$.

Case 2. $x \in\left[t_{i}, t_{i+1}\right], x+\delta \in\left[t_{j}, t_{j+1}\right], i<j$.

Then

$$
\begin{aligned}
& \left|h_{n}(x+\delta)-h_{n}(x)\right| \\
& \quad \leq\left|h_{n}\left(t_{i+1}\right)-h_{n}(x)\right|+\left|h_{n}\left(t_{j}\right)-h_{n}\left(t_{i+1}\right)\right|+\left|h_{n}(x+\delta)-h_{n}\left(t_{j}\right)\right| \\
& \quad \leq 2 \omega(h ; \delta)+\omega(h ; \delta)+2 \omega(h ; \delta)
\end{aligned}
$$

by Case 1 , monotonicity of $\omega(h ; t)$, and the fact that $h(x)=h_{n}(x)$ at the partition points $t_{k}$.

We note that (10) implies that if $h \in D T\left(N_{\delta}(\lambda)\right)$ or $D T(-1,1)$, then $h_{n} \in$ $D T\left(N_{\delta}(\lambda)\right)$ or $D T(-1,1)$, respectively.

3. Convergence Results. Our first result is on pointwise convergence in the general case. 
THEOREM 1. If for any $\lambda \in(-1,1)$ for which there exists a $\delta>0$ such that $N_{\delta}(\lambda) \subset J$, one has $k \in D T\left(N_{\delta}(\lambda)\right) \cap L_{1}(J)$ and $f \in D T\left(N_{\delta}(\lambda)\right) \cap R(J)$, then

$$
I\left(k f_{n} ; \lambda\right) \rightarrow I(k f ; \lambda) \text { as } n \rightarrow \infty .
$$

Proof. One has

$$
I\left(k f-k f_{n} ; \lambda\right)=I\left(k r_{n} ; \lambda\right)=\int_{-1}^{1} k(x) \frac{r_{n}(x)-r_{n}(\lambda)}{x-\lambda} d x+r_{n}(\lambda) I(k ; \lambda),
$$

where $I(k ; \lambda)$ exists by the hypotheses on $k$. Now, since $f \in D T\left(N_{\delta}(\lambda)\right)$, for any $\varepsilon>0$, we can choose $\eta>0$ such that $\Delta=(\lambda-\eta, \lambda+\eta) \subset N_{\delta}(\lambda)$ and

$$
\int_{\Delta}|k(x)| \frac{\omega\left(r_{n} ;|x-\lambda|\right)}{|x-\lambda|} d x \leq C \int_{\Delta} \frac{\omega(f ;|x-\lambda|)}{|x-\lambda|} d x<\varepsilon,
$$

where $C=6 \max _{x \in N_{\delta(\lambda)}}|k(x)|$. Hence, since $\left\|r_{n}\right\|=o(1)$,

$$
\begin{aligned}
\left|I\left(k r_{n} ; \lambda\right)\right| \leq & \int_{J-\Delta}|k(x)| \frac{\left|r_{n}(x)-r_{n}(\lambda)\right|}{|x-\lambda|} d x \\
& +\int_{\Delta}|k(x)| \frac{\left|r_{n}(x)-r_{n}(\lambda)\right|}{|x-\lambda|} d x+\left|r_{n}(\lambda)\right||I(k ; \lambda)| \\
& \leq\left\|r_{n}\right\|\left(2 \int_{J-\Delta} \frac{|k(x)|}{|x-\lambda|} d x+|I(k ; \lambda)|\right)+\varepsilon \\
& <2 \varepsilon \text { for } n \geq n_{0}(\varepsilon) . \quad \square
\end{aligned}
$$

Our second result deals only with the Jacobi case treated by Gerasoulis [3]. Before we state our theorem, we define two function classes which are subclasses of $D T(J)$ :

$$
\begin{aligned}
H_{\mu} & =\left\{f: \omega(f ; t) \leq M t^{\mu} \text { for some } M>0,0<\mu \leq 1\right\} \\
L_{\nu} & =\left\{f: \omega(f ; t) \leq M /\left|\log ^{\nu} t\right| \text { for } t<1 \text { and some } M>0 ; 1<\nu\right\} .
\end{aligned}
$$

THEOREM 2. Let $w(x)=(1-x)^{\alpha}(1+x)^{\beta}$ with $\gamma=\min (\alpha, \beta)>-1$. Then $I\left(w f_{n} ; \lambda\right) \rightarrow I(w f ; \lambda)$ as $n \rightarrow 0$ uniformly for all $\lambda \in(-1,1)$ if either (a) $f \in H_{\mu}$ and $\mu+\gamma>0$ or (b) $f \in L_{\nu}$ and $\gamma \geq 0$. In particular, if $f \in H_{1}$, i.e., if $f$ satisfies a Lipschitz condition, then we have uniform convergence for all $w$.

Proof. As above, we write the error term $I(w f ; \lambda)-I\left(w f_{n} ; \lambda\right)$ as

$$
\int_{-1}^{1} w(x) \frac{r_{n}(x)-r_{n}(\lambda)}{x-\lambda} d x+r_{n}(\lambda) I(w ; \lambda)
$$

We first deal with the second term and use the fact [7, Section 4.62] that in a neighborhood of $\lambda=1$

$$
I(w ; \lambda)= \begin{cases}O(1-\lambda)^{\alpha} & \text { if } \alpha \text { is not an integer, } \\ O(1-\lambda)^{\alpha}|\log (1-\lambda)| & \text { if } \alpha \text { is an integer }\end{cases}
$$

and similarly, in a neighborhood of $\lambda=-1$

$$
I(w ; \lambda)= \begin{cases}O(1+\lambda)^{\beta} & \text { if } \beta \text { is not an integer, } \\ O(1+\lambda)^{\beta}|\log (1+\lambda)| & \text { if } \beta \text { is an integer }\end{cases}
$$


while $I(w ; \lambda)=O(1)$ in $[-1+s, 1-s]$ for any $s>0$. Since $r_{n}(1)=0,\left|r_{n}(\lambda)\right| \leq$ $\omega\left(r_{n} ; 1-\lambda\right) \leq 6 \omega(f ; 1-\lambda)$, and we have that in $[1-s, 1]$, if $\alpha$ is not an integer,

$$
\left|r_{n}(\lambda) I(w ; \lambda)\right| \leq \begin{cases}K(1-\lambda)^{\alpha} M(1-\lambda)^{\mu} & \text { if (a) holds, } \\ K(1-\lambda)^{\alpha} M /\left|\log ^{\nu}(1-\lambda)\right| & \text { if (b) holds }\end{cases}
$$

while, if $\alpha$ is an integer, the right-hand sides must be multiplied by $|\log (1-\lambda)|$. In either case, we see that we can make $\left|r_{n}(\lambda) I(w ; \lambda)\right|<\varepsilon$ for $\lambda \in[1-s, 1]$ by choosing $s$ sufficiently small. Similarly, we can make

$$
\left|r_{n}(\lambda) I(w ; \lambda)\right|<\varepsilon \quad \text { for } \lambda \in[-1,-1+s] .
$$

Finally, for $\lambda \in[-1+s, 1-s]$,

$$
\left|r_{n}(\lambda) I(w ; \lambda)\right|=O\left(r_{n}(\lambda)\right)=o(1),
$$

so that given any $\varepsilon>0$, there exists $n_{0}=n_{0}(\varepsilon)$ such that for all $\lambda \in(-1,1)$ and all $n \geq n_{0},\left|r_{n}(\lambda) I(w ; \lambda)\right|<\varepsilon$.

We now write the integral

$$
\int_{-1}^{1} w(x) \frac{r_{n}(x)-r_{n}(\lambda)}{x-\lambda} d x
$$

as the sum of three integrals,

$$
\int_{U}+\int_{\substack{|x-\lambda| \geq D_{n} \\ x \notin U}}+\int_{\substack{|x-\lambda| \leq D_{n} \\ x \notin U}},
$$

where $U=[-1,-1+r] \cup[1-r, 1]$ for some $r$ to be chosen below. For the first integral, we have that

$$
\begin{aligned}
& \left|\int_{-1}^{-1+r} w(x) \frac{r_{n}(x)-r_{n}(\lambda)}{x-\lambda} d x\right| \\
& \leq C_{1} \int_{-1}^{-1+r}(1+x)^{\beta}|x-\lambda|^{\mu-1} d x \\
& \leq C_{2} \int_{-1}^{-1+r}(1+x)^{\beta+\mu-1} d x<\varepsilon \text { for some } r \text { if (a) holds, } \\
& \left|\int_{-1}^{-1+r} w(x) \frac{r_{n}(x)-r_{n}(\lambda)}{x-\lambda} d x\right| \\
& \leq C_{3} \int_{-1}^{-1+r}\left|\log ^{-\nu}(1+x)\right| d x<\varepsilon \quad \text { for some } r \text { if (b) holds. }
\end{aligned}
$$

Similarly, $\left|\int_{1-r}^{1}\right|<\varepsilon$ for some $r$ if (a) or (b) holds. Hence, we can choose an $r>0$ such that $\int_{U}<2 \varepsilon$. For the second integral,

$$
\begin{aligned}
\left|\int_{\substack{|x-\lambda| \geq D_{n} \\
x \notin U}} w(x) \frac{r_{n}(x)-r_{n}(\lambda)}{x-\lambda} d x\right| \\
\leq \max _{-1+r \leq x \leq 1-r} w(x) \cdot 2\left\|r_{n}\right\| \cdot \int_{\substack{|x-\lambda| \geq D_{n} \\
x \notin U}} \frac{1}{|x-\lambda|} d x \\
\leq K\left\|r_{n}\right\| \log D_{n} \mid=o(1) \quad \text { since }\left\|r_{n}\right\| \leq \omega\left(f ; D_{n}\right) .
\end{aligned}
$$


Finally,

$$
\left|\int_{\substack{|x-\lambda| \leq D_{n} \\ x \notin U}} w(x) \frac{r_{n}(x)-r_{n}(\lambda)}{x-\lambda} d x\right| \leq K_{1} \int_{\substack{|x-\lambda| \leq D_{n} \\ x \notin U}} \frac{\omega(f ;|x-\lambda|)}{|x-\lambda|} d x=o(1)
$$

independent of $\lambda$ if (a) or (b) holds. This completes the proof.

We now consider a second approach based on (4) in which we approximate $g_{\lambda}$ by the piecewise linear function $g_{\lambda n}$, so that the approximation to $I(k f ; \lambda)$ is given by

$$
\int_{-1}^{1} k(x) g_{\lambda n}(x) d x+f(\lambda) I(k ; \lambda)
$$

and the error in the approximation, $E_{n}(\lambda)$, is given by

$$
E_{n}(\lambda)=\int_{-1}^{1} k(x)\left(g_{\lambda}(x)-g_{\lambda n}(x)\right) d x
$$

We note that if $k \in D T\left(N_{\delta}(\lambda)\right)$, then $I(k ; \lambda)$ is finite and can either be found in a table of Hilbert transforms, e.g. [2], or evaluated numerically as

$$
I(k ; \lambda)=\int_{-1}^{\lambda} \frac{k(x)-k(\lambda)}{x-\lambda} d x+\int_{\lambda}^{1} \frac{k(x)-k(\lambda)}{x-\lambda} d x+k(\lambda) \log \frac{1-\lambda}{1+\lambda} .
$$

We shall say that a function $f \in D(I)$ for a closed interval $I$ if $f^{\prime}(x)$ exists for every $x$ in the interior of $I$ and the right-hand and left-hand derivatives exist at the left and right endpoints, respectively. If $f \in D(J)$, then $g(x ; \lambda)$ is uniformly continuous for all pairs $(x ; \lambda) \in J \times J$. We now state two theorems on the convergence of $E_{n}(\lambda)$ to zero as $n \rightarrow \infty$, one on pointwise convergence and one on uniform convergence.

THEOREM 3. If for some $\lambda \in(-1,1), k \in L_{1}(J)$ and $f \in H_{1}\left(N_{\delta}(\lambda)\right) \cap R(J)$, then $E_{n}(\lambda) \rightarrow 0$ as $n \rightarrow \infty$.

Proof. If $f \in H_{1}\left(N_{\delta}(\lambda)\right) \cap R(J)$, then $g_{\lambda} \in R(J)$, and we can apply Theorem 1 in $[5$, p. 6].

THEOREM 4. If $k \in L_{1}(J)$ and $f \in D(J)$, then $E_{n}(\lambda) \rightarrow 0$ as $n \rightarrow \infty$ uniformly in $\lambda$. Hence, if $k \in D T(-1,1) \cap L_{1}(J)$ and $f \in D(J)$, then (13) converges uniformly to $I(k f ; \lambda)$.

Proof. For any $\lambda,\left|r_{n}(x ; \lambda)\right|=\left|g_{\lambda}(x)-g_{n \lambda}(x)\right| \leq \omega\left(g_{\lambda} ; D_{n}\right)$. But $\omega\left(g_{\lambda} ; D_{n}\right)$ is independent of $\lambda$ by the uniform continuity of $g(x ; \lambda)$ in $\lambda$. Hence

$$
\left|E_{n}(\lambda)\right| \leq\left\|r_{n}(x ; \lambda)\right\| \int_{-1}^{1}|k(x)| d x=o(1) \text { uniformly in } \lambda .
$$

If $k \in D T(-1,1)$, then $I(k ; \lambda)$ exists for all $\lambda \in(-1,1)$, which yields the uniform convergence of (13).

Remark. The two approaches (12) and (13) correspond to the two methods for evaluating $I(w f ; \lambda)$ based on interpolation at the zeros of the Jacobi polynomial $P_{n}^{(\alpha, \beta)}(x)$, and analogous results exist for pointwise convergence. See [1, Theorem $2.1]$ and $[4$, Theorem $7(1)]$. In both situations, the more accurate approach requires more stringent conditions on $f$ to insure pointwise convergence. 
1. G. CRiscuolo \& G. MASTROiAnNi, "On the convergence of an interpolatory product rule for evaluating Cauchy principal value integrals," Math. Comp., v. 48, 1987, pp. 725-735.

2. A. ERdélyi, W. Magnus, F. Oberhettinger \& F. G. Tricomi, Tables of Integral Transforms, vol. II, McGraw-Hill, New York, 1954.

3. A. GerasoUlis, "Piecewise-polynomial quadratures for Cauchy singular integrals," SIAM J. Numer. Anal., v. 23, 1986, pp. 891-902.

4. P. RABINOWITZ, "Numerical integration in the presence of an interior singularity," J. Comput. Appl. Math., v. 17, 1987, pp. 31-41.

5. P. RABINOWITZ, "The convergence of noninterpolatory product integration rules," in $\mathrm{Nu}$ merical Integration (P. Keast and G. Fairweather, eds.), Reidel, Dordrecht, 1987, pp. 1-16.

6. C. StewART, "On the numerical evaluation of singular integrals of Cauchy type," J. Soc. Indust. Appl. Math., v. 8, 1960, pp. 342-353.

7. G. Szegö, Orthogonal Polynomials, Amer. Math. Soc. Colloq. Publ., vol. 23, Amer. Math. Soc., Providence, R.I., 1975. 